\title{
Comparison of two protocols of periosteal distraction osteogenesis in a rabbit calvaria model
}

\author{
Nikola Saulacic, ${ }^{1,2}$ Ken Nakahara, Tateyuki lizuka, ${ }^{1}$ Maiko Haga-Tsujimura, ${ }^{1}$ Willy Hofstetter, ${ }^{3}$ \\ Paolo Scolozzi ${ }^{2}$
}

\footnotetext{
${ }^{1}$ Department of Cranio-Maxillofacial Surgery, Inselspital, Bern University Hospital, and University of Bern, Switzerland;

${ }^{2}$ Service of Maxillofacial and Oral Surgery, Department of Surgery, University of Geneva Hospitals, Switzerland

${ }^{3}$ Bone Biology \& Orthopaedic Research, Department Clinical Research, University of Bern, Switzerland
}

Correspondence to: N. Saulacic; Department of Cranio-Maxillofacial Surgery, Bern University Hospital and University of Bern, CH-3010 Bern, Switzerland; Tel.: +4131632 35 63; Fax: +41382 02 79; e-mail: nikola.saulacic@insel.ch

Running head: Periosteal Distraction Osteogenesis 


\section{ABSTRACT}

The regenerative pathways during periosteal distraction osteogenesis may be influenced by the local environment composed by cells, growth factors, nutrition and mechanical load. The aim of the present study was to evaluate the influence of two protocols of periosteal distraction on bone formation. Custom made distraction devices were surgically fixed onto the calvariae of 60 rabbits. After an initial healing period of 7 days, two groups of animals were submitted to distraction rates of 0.25 and $0.5 \mathrm{~mm} / 24 \mathrm{~h}$ for 10 days, respectively. Six animals per group were sacrificed 10 (middistraction), 17 (end-distraction), 24 (1-week consolidation), 31 (2-week consolidation) and 77 days (2-month consolidation) after surgery. Newly formed bone was assessed by means of micro-CT and histologically. Expression of transcripts encoding tissue-specific genes (BMP-2, RUNX2, ACP5, SPARC, collagen I $\alpha 1$, collagen II $\alpha 1$ and SOX9) was analyzed by quantitative PCR. Two patterns of bone formation were observed, originating from the old bone surface in Group I and from the periosteum in Group II. Bone volume and bone mineral density significantly increased up to the 2month consolidation period within the groups $(p<0.05)$. Significantly more bone was observed in Group II compared to Group I at the 2-month consolidation period $(p<0.001)$. Expression of transcripts encoding osteogenic genes in bone depended on the time-point of observation $(p<0.05)$. Low level of transcripts reveals an indirect role of periosteum in the osteogenic process. Two protocols of periosteal distraction in the present model resulted in moderate differences in terms of bone formation.

Key Words: animal model, bone, periosteum, distraction osteogenesis, histology, micro-CT, gene expression. 


\section{INTRODUCTION}

Distraction osteogenesis (DO) is a technique of gradual elongation of the bone fragments within the space created by osteotomy that results in formation of hard and soft tissues. ${ }^{1}$ The principle of DO was subsequently introduced in the cranio-maxillofacial region, ${ }^{2}$ using the protocols similar to those developed for long-bone distraction. ${ }^{3}$ Ilizarov originally reported that an increase in the daily rhythm of distraction yields better bone formation as compared to one single activation in conventional $\mathrm{DO} .{ }^{4}$ Fractionated distraction protocols are associated with significantly less injury to nerves, blood vessels, periosteum and skin. Nevertheless, variations in the protocols of distraction differently influenced the success of mandibular DO depending on the model used. ${ }^{5-7}$

Subtle differences in bone formation induced by differing distraction rates may be noticed at the molecular level rather than histologically. ${ }^{8} \mathrm{~A}$ decrease in bone formation was associated with a decrease in the synthesis of bone-specific extra-cellular matrix (ECM) proteins induced by hyperphysiological strains of distraction ${ }^{9}$ or acute mandibular lengthening. ${ }^{10}$ Standard rate of mandibular distraction stimulated different expression of BMP-2 and BMP-4 in comparison to fracture healing ${ }^{11,12}$ or rapid rate of distraction. ${ }^{13}$

Compared to conventional DO, the distraction gap formed by periosteal distraction osteogenesis (PDO) is bordered by the original, intact surface of the bone base and by the periosteal (i.e., cambial) layer. Under certain indications, the need for performing an osteotomy and its associated difficulties might be avoided. Strains tending to pull the periosteum away from the bone are typically osteogenic, but it is quite difficult to produce controlled loads on periosteum in vivo. ${ }^{14}$ Several animal studies have reported on the characteristics of the bone formation following PDO. ${ }^{15-21}$ However, variations exist that are likely due to use of different animal models, sites and distraction devices and the total amount of distraction performed. Recent investigations compared distraction regenerate in PDO with immediate elevation of the periosteum. Comparable amounts and quality of new bone were achieved during a 45-day period of consolidation by static or dynamic periostal distraction on the calvarial bone of miniature pigs. ${ }^{22,23}$ In contrast, Claes at al. ${ }^{24}$ found significantly more osteoid and bone marrow with lateral elevation of a hydroxyapatite-coated titanium mesh in the tibia of sheep when compared to immediate elevation. It is, however, unclear whether the manipulation of distraction rate may affect the formation of new bone during PDO.

We hypothesized that the applied parameters of PDO may influence the nature and kinetics of bone formation. Two protocols of periosteal distraction were thus performed to compare: (i) 
formation and origin of new bone and (ii) molecular events characterizing the bone formation over time.

\section{MATERIAL \& METHODS}

For the experiment were used 60 adult, female New Zealand rabbits with an average weight of approximately $3 \mathrm{~kg}$. Animals were housed in the Central Animal Facility of the University of Bern with an adjusted climate (temperature $22-24^{\circ} \mathrm{C} \pm 2{ }^{\circ} \mathrm{C}$, humidity $30-60 \% \pm 5 \%$, a light:dark cycle of 12:12 hours), without excessive or startling noises and with standard diet and water ad libitum. The protocol was approved by the Committee for Animal Research, State of Bern, Switzerland (Approval No. 14/11).

\section{Surgical procedure}

The surgeries were performed at the Experimental Surgery Unit, Department of Clinical Research and Clinic for Large Animals, Bern University Hospital. Premedication included Ketamin 65 mg/kg (Vétoquinol AG, Bern, Switzerland) and Xylazin $4 \mathrm{mg} / \mathrm{kg}$ s.c. (Vétoquinol AG, Bern, Switzerland) in neck wrinkle (pain free). Narcosis was maintained with Ketamin 130 mg/kg (Vétoquinol AG, Bern, Switzerland) and Xylazin $8 \mathrm{mg} / \mathrm{kg}$ in $100 \mathrm{ml} \mathrm{NaCl}$ i.v. (Vétoquinol AG, Bern, Switzerland) under spontaneous breading of $\mathrm{O}_{2}$ by the mask. Intraoperative analgesia was achieved with Fentanyl plaster 2.1 mg (Janssen_Cilag AG, Baar, Switzerland) and local anaesthesia in the operation area using articain (40 mg) and adrenalin $5 \mu \mathrm{g}$ (Sanofi-Avensis SA, Vernier, Switzerland) with a continuous effect for 3 days.

The study was designed as a prospective, controlled experimental study. Two groups of 30 animals with five healing periods were established to assess the effects of different distraction protocols (Fig. 1). During the surgical intervention, one distraction device was placed on the calvaria of each rabbit. Using an aseptic technique (shaving of the operative area and disinfection with betadine), a midsagittal incision was made through the skin and the periosteum. Both the skin and the periosteal flaps were carefully reflected from the forehead to expose the calvarial bone on both sides of the midline. In each rabbit, the custom made distraction device (Synthes $\mathrm{GmbH}$, Oberdorf, Switzerland) was fixed with 4 micro screws (Medartis AG, Basel, Switzerland) on the calvarium bone (Fig. 2A). The experimental device has a distraction mesh with dimensions of $10 \times 12 \mathrm{~mm}$. Prior to placement, the distraction mesh was addapted to the curvature of the calvarial bone. The periosteum 
and skin were closed in two layers (Fig. 2B, C). Following surgery, rabbits were observed until they were completely recovered and then transferred to cages.

All animals were left for a healing period of 7 days (latency period). The periosteal distraction was performed at $0.25 \mathrm{~mm} / 24 \mathrm{~h}$ (Group I) or $0.5 \mathrm{~mm} / 24 \mathrm{~h}$ (Group II) for 10 days to achieve the total amount of augmentation of 2.5 and $5 \mathrm{~mm}$, respectively. Six animals of each group were sacrificed at day $10^{\text {th }}$ (mid-distraction), $17^{\text {th }}$ (end-distraction), $24^{\text {th }}$ days (1-week consolidation), $38^{\text {th }}$ days $(2$-week consolidation) and $77^{\text {th }}$ (2-month consolidation) after surgery. The euthanasia was performed following premedication with Ketamin $65 \mathrm{mg} / \mathrm{kg}$ (Vétoquinol AG, Bern, Switzerland) and Xylazin 4 $\mathrm{mg} / \mathrm{kg}$ i.v. (Vétoquinol AG, Bern, Switzerland) in the neck wrinkle. After the animal was asleep, pentobarbital $120 \mathrm{mg} / \mathrm{kg}$ i.v. (Streuli Pharma AG, Uznach, Switzerland) was injected. The calvariae of rabbits were block-resected using an oscillating autopsy saw. The specimens of three animals were processed for the histological and micro-CT analysis and from another three animals for the quantitative PCR.

\section{Histological analysis}

Prior to histologic preparation, the recovered segments were fixed in $4 \%$ buffered formalin combined with $1 \% \mathrm{CaCl}_{2}$ for at least $48 \mathrm{~h}$ at ambient temperature. The specimens were processed for the production of undecalcified ground sections as described by Schenk et al. ${ }^{25}$ Briefly, the samples were rinsed in running tap water, dehydrated in ascending concentrations of ethanol and embedded in methylmethacrylate. The embedded tissue blocks were cut along the axis of the distraction device into approximately $400 \mu \mathrm{m}$-thick ground section using a slow-speed diamond saw Varicut ${ }^{\circledR}$ VC-50 (Leco, Munich, Germany). After mounting the sections onto acrylic glass slabs, they were ground and polished to a final thickness of about $100 \mu \mathrm{m}$ and surface stained with basic fuchsin and toluidine blue/McNeal. Digital photography was performed using a Nikon DS-Ri1 ${ }^{\circledR}$ digital camera connected to a Nikon Eclipse $\mathrm{E} 800^{\circledR}$ microscope (Nikon Corporation, Tokyo, Japan).

\section{Micro-CT analysis}

The distraction sites were subjected to radiography ( $25 \mathrm{kVP}$ for $10 \mathrm{sec}$.) in two projections using a desktop Cone-Beam scanner ( $\mu \mathrm{CT}$ 40, Scanco Medical AG, Brüttisellen, Switzerland). The X-ray source $(\mathrm{E})$ was set at $70 \mathrm{kVp}$ with $114 \mathrm{~mA}$ at high resolution (1000 projections $\left./ 180^{\circ}\right)$, which showed an image matrix of $2048 \times 2048$ pixels. The diameter of the sample holder was $30.7 \mathrm{~mm}$, which 
allowed an increment (Resolution) of $15 \mu \mathrm{m}$ (=Voxelsize). Integration time was set on 3s. The microCT slices (700) were reconstructed perpendicular to the saggital axis of the calvarium. The region of new bone within the distraction gap was selected manually, between the old bone surface and the distraction mesh. The evaluation of the reconstructed 2D images was made with 3D Segmentation of Volume of Interest, Gauss Sigma at 0.8 and Gauss support at 1 . Bone volume (BV, $\mathrm{mm}^{3}$ ) and bone mineral density (BMD, $\mathrm{mg} \mathrm{HA} / \mathrm{mm}^{3}$ ) were determined.

\section{Real-time PCR}

Three samples were collected from each site: (i), periosteum (ii) soft tissue and (iii) bone, composed of old and new bone. The skin was elevated from the head and three samples collected from each site. The periosteum was incised at the base of the distraction gap and carefully removed from the distraction mesh (first sample). Thereafter, the device was removed. The complete bone thickness corresponding to the gap region was excised from the calvaria using the oscillating saw. The soft tissue underneath the mesh was separated form the underlying bone (second sample). The new bone could be not divided precisely from the old bone and thus used as a single bone fragment for the analysis (third sample). Subsequently, the samples were stored in RNAlater (Qiagen, Basel, Switzerland) at $-70^{\circ} \mathrm{C}$ until use. The weight of each sample was determined and was fragmented in cryo tubes in liquid nitrogen.

Total RNA was prepared using an RNeasy Mini Kit (Qiagen, Basel, Switzerland), following the instructions of the manufacturer. RNA was quantified with a NanoDrop 2000 spectrophotometer (Thermo Scientific, Wilmington, DE). RNA quality was assessed in a Bioanalyser 2100 (Agilent Technologies, Santa Clara, CA). For PCR, total RNA was reverse-transcribed using MLV reverse transcriptase (Promega, Dübendorf, Switzerland). To analyze the gene expression within the repair tissues, quantitative RT-PCR was performed, using pre-synthesized Assays-on-Demand (AoD, Life Technologies/ABI, Zug, Switzerland) for BMP-2 (P121209-003G12), ACP5 (P121209-003H03), RUNX2 (P121209-003H01), SPARC (P121209-003G11), collagen I a1 (P121209-003G09), collagen II a1 (P121209-003G10) and SOX9 (P121209-003H02). PCR was performed on 7500 Fast RealTime PCR System ${ }^{\#}$ and the data was evaluated using the sequence detection software SDS v2.0.1 (Life Technologies, Grand Island, NY). 


\section{Statistical analysis}

A multivariate t-test (Tukey's test) was used to compare differences in BV, BMD and expression of transcripts within the groups throughout the observation period and between the groups at the same time point. A significance level of 0.05 was chosen to determine statistical significance. The statistical analysis was processed using SPSS for Windows Release 19.0, standard version (IBM SPSS, Chicago, IL).

\section{RESULTS}

\section{Qualitative histological analysis}

All 60 animals survived the surgical procedures without complications. During the observation period, clinical inspection did not reveal dehiscences or signs of infection at the surgical sites. Old bone, consisting of a tabula externa and interna with intervening marrow cavities were recognizable in the micro-CT images and histologically (Figs. 3 and 4). Newly formed bone in the distraction gap was observed in all animals. The process of bone formation was comparable within the groups for all time-periods, but not between the groups. The type of new bone in Group I was always primary, new bone at the leading edge of bone apposition. New bone was found arising from the periosteum in Group II, with almost no bone formation in the middle of the distraction gap. During the consolidation period, signs of bone turnover were observed in both groups. Bone cavities in Group II were more enlarged than in the Group I, and opened towards the center of the distraction gap. The height of the new bone was asymmetrical within the distraction gap in both groups, but was always greater in Group II.

\section{Distraction period}

Bone apposition and bone resorption were observed in all animals (Figs. 3 and 4). Osteoblasts and osteoid were seen at the apposition sites and osteoclasts at the resorption sites. Bone cavities with immature bone marrow were associated with calvarial bone marrow. The amount of new bone increased from the mid-distraction to the end-distraction period. Non-uniform deposition of a new, primary bone over the old bone surface was observed in Group I at the mid-distraction and enddistraction period (Fig. 3). The distraction gap between the new bone and the mesh was occupied by a vascularized, loose connective tissue. New bone in Group II originated from the periosteum, with fine trabeculae of woven bone elongated parallel to the distraction vector (Fig. 4). In the center of the 
distraction device, a discernible periosteal layer was occasionally missing. Outside the distraction device, a moderately thick and almost uniform layer of new bone was observed in both groups. For all samples, the thickness of the new bone decreased with increasing the distance from the mesh.

\section{Consolidation period}

Features of bone formation during the early-consolidation period in the micro-CT images corresponded to the observations made in the histological sections (Figs. 3 and 4). No major differences were observed between 1-week and 2-week consolidation periods within the groups. Islets of new, primary bone reinforced by parallel-fibered bone within the immature bone marrow were seen in both groups. Large bone marrow cavities in Group I were vascularized (Fig. 3). Osteoid and osteoblasts indicative of ongoing bone apposition were observed at the leading edge of bone formation. The surface of the new bone deprived of periosteum in Group II was irregular (Fig. 4). At the periphery of the distraction device, the surface contour of new bone in both groups was more flat.

Total bone thickness obtained by micro-CT at the 2-month consolidation period matched that seen in the ground sections (Figs. 3 and 4). The maximum height of the new bone in Group II (Fig. 4) by far exceeded that observed in Group I (Fig. 3), and was even seen penetrating through the perforation holes of the distraction mesh. Primary bone was gradually replaced by lamellar bone in all animals and the border between old and new bone was not distinguishable. Osteoid and osteoblasts were present at various sites of the new bone. In both groups, fat tissue was present within the new bone cavities and in the distraction gap, between new bone and the distraction mesh. Peripheral to the distraction device, compact bone with bone marrow cavities was found in both groups. Signs of bone resorbtion were not observed at the peripheral sections of the samples.

\section{Micro-CT analysis}

The BV and BMD for both groups throughout the observation period are shown in Table 1. The BV in Group I increased up to the 1-week consolidation period and then decreased. In comparison to the mid-distraction period, significantly more BV was found at the 1-week $(p=0.001)$, 2-week $(p=0.010)$ and 2-month $(p=0.046)$ consolidation periods. Furthermore, the 1-week consolidation period demonstrated significantly more $\mathrm{BV}$ in comparison to the end-distraction period $(p=0.012)$. The volume of new bone in Group II increased throughout the observation time, especially from the 2week to 2-month consolidation period. Significantly more BV was found at the 2-week consolidation 
period in comparison to the mid-distraction period $(p=0.010)$ and at 2-month consolidation period compared to all other time points $(p<0.001)$. The difference in $\mathrm{BV}$ between the two groups was found at the 2-month consolidation period, with a three-fold increase in Group II in comparison to Group I $(p<0.001)$.

The value of BMD gradually increased over time in both groups. Significant differences were found from the mid-distraction to the 2-month consolidation period in Group I ( $p=0.007)$ and Group II $(p=0.003)$ and from the end-distraction to the 2-month consolidation period in Group I $(p=0.031)$. There were no significant differences in BMD between the two groups at any time point.

\section{Real-time PCR analysis}

Analysis of the expression of transcripts in bone revealed a similar pattern in both experimental groups, independent of the distraction kinetics (Fig. 5, Table 2). The levels of transcripts were elevated during the distraction and 2-months consolidation period compared to the earlyconsolidation period. BMP-2 mRNA in Group I throughout the observation period contrasted this pattern. Levels of transcripts increased after 2 weeks of consolidation compared to mid-distraction ( $p$ $=0.004)$, end-distraction $(p=0.004)$ and 2 -months consolidation period $(p=0.006)$. In Group II, expression of transcripts encoding RUNX2 ( $p=0.012)$, SOX9 $(p=0.043)$ and SPARC $(p=0.034)$ was significantly increased at the mid-distraction period compared to the 1-week consolidation period. BMP-2 mRNA were elevated in Group I after 2 weeks of consolidation when compared to Group II at the same time point $(p=0.007)$.

The level of transcripts encoding in the soft tissue was lower in both distraction groups as compared to bone (Fig. 5, Table 3). BMP-2 mRNA was not detectable. SPARC transcript level was significantly higher in Group I at mid-distraction than at 1-week consolidation period $(p=0.016)$ and in Group II at mid-distraction than at 2-week consolidation period $(p=0.010)$. SOX9 mRNA expression in Group II was significantly higher at mid-distraction than at 2-month consolidation period $(p=0.045)$.

The levels of transcripts within the periosteum were lower in both distraction groups throughout the experiment when compared to bone (Fig. 5, Table 4). Transcripts encoding the osteoclast marker ACP5 and collagen II $\alpha 1$ were not detectable. In Group II, level of RUNX2 mRNA was increased at the mid-distraction compared to Group I at the same time-point $(p=0.023)$ and within Group II when compared to 2-months consolidation period ( $p=0.009$ ). 


\section{DISCUSSION}

Present findings support the current knowledge that the periosteal distraction can induce de novo bone formation. Two protocols of periosteal distraction were not equally efficient in the induction of new bone formation in this particular model. Histological observations corresponded to the previous findings for the given rate of PDO. ${ }^{26,27}$

Uneven bone apposition on the old bone surface with blood vessels ingrowth was previously observed in rabbits using a distraction rate of $0.25 \mathrm{~mm} / 24 \mathrm{~h}^{26}$ and in rats using a rate of $0.1 \mathrm{~mm} / 24$ h. ${ }^{28}$ Patch-wise bone formation was likely caused by the remnants of prominent coagulum, as they need to be resorbed before new bone is formed. ${ }^{21}$ The absence of bone apposition observed in Group II might be caused by the higher rate of distraction performed that impaired the interaction between periosteum and the calvarial bone, important for the new bone apposition. ${ }^{29}$ Applied distraction rate was apposite to stimulate bone formation directly from the periosteum. New cortical bone underneath the periosteum with irregular bone islets in the region of the gap was reported on the lateral surface of the rabbit's mandible using the same daily rate of distraction. ${ }^{27,30}$ Despite the observed characteristics in bone formation, the BV during early observation period depended on the given time-point and not on the rate of distraction performed. These results corroborate the previous findings on static and dynamic periosteal elevation on the calvaria of minipigs. ${ }^{22,23}$

An alternating temporal expression of EMC transcripts correlates with the new, woven bone formation during activation and lamellar bone formation during late-consolidation period. Consequently, higher SD during early-consolidation period corresponds to an increased dynamics of bone turnover from primary to the lamellar bone. Increased value of SPARC mRNA, which codes for the protein osteonectin that is secreted by osteoblasts and initiate mineralization was expected, but contrast the findings from the mandibular distraction in rabbits. ${ }^{31}$ Expression pattern of osteogenic markers in PDO might differ from conventional DO because of the absence of osteotomy-related stimuli. ${ }^{11,12}$ Possible reasons for these differences are speculative and should be determined by using more refined approaches than employed here. The statistical analysis revealed some changes in the mRNA level in bone over time, but the overall impact of the distraction rate may be considered weak. One possible reason might be an interaction between the old and new bone within the single bone fragment used for the analysis.

Up-regulation of BMP-2 by lower distraction rate at early-consolidation period corroborates the results from the mandibular distraction in rabbits. ${ }^{13,32}$ Alternating expression pattern of BMP-2 was 
previously observed in femur distraction; BMP-2 promoted vascularization in surrounding musculature during activation and concurrently, during early-consolidation period in the gap region. ${ }^{33}$ This pattern does not correspond entirely to the present results. Stress application directly to the periosteum might have altered a relationship between angiogenesis and bone formation in the periosteum and underlying bone. ${ }^{34}$

Appositional periosteum from the lateral surface of the temporal bone demonstrates high expression of RUNX2, which is a key transcription factor associated with osteoblast differentiation. ${ }^{14}$ Increased level of transcripts in periosteum at mid-distraction in Group II implies that the RUNX2 signaling may play a role in the translation of mechanical forces. ${ }^{35,36}$ Mechanical stretching generally increases the expression of all BMP-2 responsive osteogenic markers, whereas a combined stretching and BMP-2 stimulation was found more efficient on gene expression than a single treatment alone. ${ }^{37}$ Nevertheless, the in vitro findings do not necessarily translate into in vivo. The viable periosteum might be less susceptible to the stress over time because of its own growing potential. ${ }^{38}$ In the present model of PDO, the transcript level of bone-specific markers in periosteum was clearly lower compared to bone. This supports the previous findings that the periosteum is not osteogenic, but serves as a source of osteogenic cells and factors necessary for bone formation. ${ }^{39,40}$

Two months of consolidation period were necessary to demonstrate differences between applied protocols of PDO. In both groups of animals, the new and the old bone appeared as a single bone fragment. It is possible that the bone modeling in Group I started earlier than in Group II, due to the lower amount of new bone formed. ${ }^{33}$ As a consequence, BV in Group I decreased throughout the consolidation period. Formation of new bone in Group II, however, continued once the activation was ceased. This delayed the process of bone modeling in comparison to Group I. The sustained bone formation in Group II was apparently caused by the bigger size of the distraction gap and not by the rate of distraction performed. The same size of distraction gap in Group I might have been achieved by using a prolonged distraction period of 20 days. The absence of this Group, thus, limits the assessment of the present results in terms of the distraction rate. The same duration of the distraction period in two Groups was applied to evaluate the process of bone formation at a given time-point and estimate the relevance of the total distance of distraction performed. Significant increase in BV at the 2-month consolidation period in Group II contrasts the histomorphometric results from the lateral surface of the rabbit's mandible, ${ }^{16,27}$ but corroborate previous findings on the 
rat's calvaria. ${ }^{41}$ Differences in BV throughout the healing period may be influenced by the healing characteristics of the calvaria compared to the mandible. ${ }^{16}$

Time, but not the rate of distraction was important for BMD change in the present study. Increase in BMD from the end-distraction to the 2-month consolidation period was demonstrated within Group I, but not within Group II. The reason for this difference may be the reduced cortical bone thickness in Group I, which would not affect BMD. ${ }^{42}$ According to the histological findings at the 2-month consolidation period, the differences in BMD between the two groups were not expected. No significant difference was seen between static and dynamic periosteal elevation with regard to the degree of mineralization, trabecular architecture and bone density on the calvaria of minipigs. ${ }^{22,23}$ New bone would possibly be more calcified by decortication of the original bone. ${ }^{17}$ This was, however, not performed as it might have overridden the effect of the periosteum stimulation alone.

High content of interstitial fat tissue was observed in both groups of animals at the 2-month consolidation period in the present study, as well as in the previous studies on PDO in rabbits. ${ }^{16,18,27}$ On the contrary, fat tissue formation has not been observed in rats even when the contribution of periosteum was deliberately severed. ${ }^{28} \mathrm{~A}$ compartment-specific anabolic response has been noticed on the periosteal progenitors from frontal and parietal bone; osteoblastic and adipogenic differentiation in these populations was influenced by embryonic lineage and developmental origin. ${ }^{43}$ Yoshiko et al. ${ }^{44}$ identified a subset of immature calvaria-derived osteoblasts that may exhibit osteoadipogenic bipotentiality, with concomitant up-regulation of RUNX2 and down-regulation of SOX9. Fat tissue formation in PDO might have significant clinical implications and its origin should be determined in the future studies.

The absence of cartilage formation in the present study contrasts the results from mandibular distraction in rabbits. ${ }^{45,46}$ Expression of collagen II $\alpha 1$ mRNA in bone and soft tissue apparently varied during observation period in both groups, but was negligible compared to collagen I $\alpha 1$. This opposes the findings in mandibular DO. ${ }^{45}$ The difference between two models of distraction is not unexpected, as released periosteum of calvaria shows neither potential for chondrogenesis nor for collagen II and SOX9 expression. ${ }^{47}$

In conclusion, two rates of periosteal distraction in the present study induced bone formation. The major effect of periosteal distraction in terms of transcripts level was found in bone. This implies that periosteum plays an indirect role in the osteogenic process during PDO. The overall impact of the applied distraction rate on $\mathrm{BV}$ at the given time-point has to be considered moderate, overridden by 
the total amount of distraction performed. This study was designed with sufficient statistical power to interpret the tendencies, using a clinically analogous model. Thus, from the clinician perspective, the size of distraction gap is of primary importance. A higher distraction rate may enhance bone formation from the periosteum, but the risk of wound dehiscence and device exposure should be carefully considered. Incomplete bone filling succeeded by using this demanding model was presumed. The new bone should not repair the original bone defect, but take place where it has never existed before. The use of exogenous growth factors in more challenging cases has been successful, but their effectiveness usually requires sustained delivery and large doses of active proteins, particularly in humans. ${ }^{48,49}$ Induction of endogenous BMP-2 by PDO might represent a more efficient and physiologic osteogenic response than an exogenous delivery. ${ }^{50}$ Further research is thus warrant to develop treatment modalities specifically targeting adult periosteum and enhance the process of bone repair and regeneration.

\section{ACKNOWLEDGEMENTS}

The authors wish to thank the staff at the Experimental Surgical Unit, Bern University Hospital for excellent handling of the animals, Mr. David Reist for the histologic preparation of the specimens, Mr. Mark Siegrist for his professional assistance during micro-CT evaluation and Ms. Silvia Dolder and Ms. Lea Hirzberger for the molecular analyses. This study was founded by Swiss Dental Association (SSO), Bern, Switzerland (Nr. 260-11). The distraction devices were generously provided by Synthes $\mathrm{GmbH}$, Oberdorf, Switzerland. 


\section{REFERENCES}

1. Ilizarov GA. The tension-stress effect on the genesis and growth of tissues. Part I. The influence of stability of fixation and soft-tissue preservation. Clin Orthop Relat Res 1989:249-281.

2. McAllister BS. Histologic and radiographic evidence of vertical ridge augmentation utilizing distraction osteogenesis: 10 consecutively placed distractors. J Periodontol 2001;72:1767-1779.

3. Saulacic N, lizuka T, Martin MS, Garcia AG. Alveolar distraction osteogenesis: a systematic review. Int J Oral Maxillofac Surg 2008;37:1-7.

4. Ilizarov GA. The tension-stress effect on the genesis and growth of tissues: Part II. The influence of the rate and frequency of distraction. Clin Orthop Relat Res 1989:263-285.

5. Stewart KJ, Lvoff GO, White SA, Bonar SF, Walsh WR, Smart RC, Poole MD. Mandibular distraction osteogenesis: a comparison of distraction rates in the rabbit model. $\mathrm{J}$ Craniomaxillofac Surg 1998;26:43-49.

6. Paccione MF, Mehrara BJ, Warren SM, Greenwald JA, Spector JA, Luchs JS, Longaker MT. Rat mandibular distraction osteogenesis: latency, rate, and rhythm determine the adaptive response. J Craniofac Surg 2001;12:175-182.

7. Hasse AR, Porksen M, Zimmermann CE. Bilateral mandibular distraction in adult dogs with an epiperiosteal distractor. Br J Oral Maxillofac Surg 2005;43:105-112.

8. Choi IH, Shim JS, Seong SC, Lee MC, Song KY, Park SC, Chung CY, Cho TJ, Lee DY. Effect of the distraction rate on the activity of the osteoblast lineage in distraction osteogenesis of rat's tibia. Immunostaining study of the proliferating cell nuclear antigen, osteocalcin, and transglutaminase C. Bull Hosp Jt Dis 1997;56:34-40.

9. Meyer U, Meyer T, Vosshans J, Joos U. Decreased expression of osteocalcin and osteonectin in relation to high strains and decreased mineralization in mandibular distraction osteogenesis. J Craniomaxillofac Surg 1999;27:222-227.

10. Warren SM, Mehrara BJ, Steinbrech DS, Paccione MF, Greenwald JA, Spector JA, Longaker MT. Rat mandibular distraction osteogenesis: part III. Gradual distraction versus acute lengthening. Plast Reconstr Surg 2001;107:441-453.

11. Ai-Aql ZS, Alagl AS, Graves DT, Gerstenfeld LC, Einhorn TA. Molecular mechanisms controlling bone formation during fracture healing and distraction osteogenesis. J Dent Res 2008;87:107118. 
12. Khanal A, Yoshioka I, Tominaga K, Furuta N, Habu M, Fukuda J. The BMP signaling and its Smads in mandibular distraction osteogenesis. Oral Dis 2008;14:347-355.

13. Cheung LK, Zheng LW, Ma L. Effect of distraction rates on expression of bone morphogenetic proteins in rabbit mandibular distraction osteogenesis. J Craniomaxillofac Surg 2006;34:263269.

14. Ochareon $\mathrm{P}$, Herring SW. Cell replication in craniofacial periosteum: appositional vs. resorptive sites. J Anat 2011;218:285-297.

15. Schmidt BL, Kung L, Jones C, Casap N. Induced osteogenesis by periosteal distraction. J Oral Maxillofac Surg 2002;60:1170-1175.

16. Sencimen M, Aydintug YS, Ortakoglu K, Karslioglu Y, Gunhan O, Gunaydin Y. Histomorphometrical analysis of new bone obtained by distraction osteogenesis and osteogenesis by periosteal distraction in rabbits. Int J Oral Maxillofac Surg 2007;36:235-242.

17. Oda T, Kinoshita K, Ueda M. Effects of cortical bone perforation on periosteal distraction: an experimental study in the rabbit mandible. J Oral Maxillofac Surg 2009;67:1478-1485.

18. Sato K, Haruyama N, Shimizu Y, Hara J, Kawamura H. Osteogenesis by gradually expanding the interface between bone surface and periosteum enhanced by bone marrow stem cell administration in rabbits. Oral Surg Oral Med Oral Pathol Oral Radiol Endod 2010;110:32-40.

19. Zakaria O, Kon K, Kasugai S. Evaluation of a biodegradable novel periosteal distractor. J Biomed Mater Res B Appl Biomater 2012;100:882-889.

20. Yamauchi K, Takahashi T, Tanaka K, Nogami S, Kaneuji T, Kanetaka H, Miyazaki T, Lethaus B, Kessler P. Self-activated mesh device using shape memory alloy for periosteal expansion osteogenesis. J Biomed Mater Res B Appl Biomater 2013;101:736-742.

21. Saulacic N, Schaller B, lizuka T, Buser D, Hug C, Bosshardt DD. Analysis of new bone formation induced by periosteal distraction in a rat calvarium model. Clin Implant Dent Relat Res 2013;15:283-291.

22. Tudor C, Bumiller L, Birkholz T, Stockmann P, Wiltfang J, Kessler P. Static and dynamic periosteal elevation: a pilot study in a pig model. Int J Oral Maxillofac Surg 2010;39:897-903.

23. Lethaus B, Tudor C, Bumiller L, Birkholz T, Wiltfang J, Kessler P. Guided bone regeneration: dynamic procedures versus static shielding in an animal model. J Biomed Mater Res B Appl Biomater 2010;95:126-130. 
24. Claes L, Veeser A, Gockelmann M, Horvath D, Durselen L, Ignatius A. A novel method for lateral callus distraction and its importance for the mechano-biology of bone formation. Bone 2010;47:712-717.

25. Schenk RK, Olah, A.J., Hermann, W Preparation of calcified tissue for light microscopy. In: Dickson, G.R., ed. Methods of calcified tissue preparation, 1st edition Amsterdam: Elsevier Science Publishers BV, 1984:1-56.

26. Estrada JI, Saulacic N, Vazquez L, Lombardi T, Ramirez JU, Bernard JP. Periosteal distraction osteogenesis: preliminary experimental evaluation in rabbits and dogs. $\mathrm{Br} \mathrm{J}$ Oral Maxillofac Surg 2007;45:402-405.

27. Altug HA, Aydintug YS, Sencimen M, Gunhan O, Ortakoglu K, Bayar GR, Dogan N. Histomorphometric analysis of different latency periods effect on new bone obtained by periosteal distraction: an experimental study in the rabbit model. Oral Surg Oral Med Oral Pathol Oral Radiol Endod 2011;111:539-546.

28. Saulacic N, Hug C, Bosshardt DD, Schaller B, Buser D, Haeniwa H, lizuka T. Relative contributions of osteogenic tissues to new bone formation in periosteal distraction osteogenesis: histological and histomorphometrical evaluation in a rat calvaria. Clin Implant Dent Relat Res 2013;15:692-706.

29. Canalis RF, Burstein FD. Osteogenesis in vascularized periosteum. Interactions with underlying bone. Arch Otolaryngol 1985;111:511-516.

30. Bayar GR, Gunaydin Y, Ortakoglu K, Gunhan O, Aydintug YS, Sencimen M. Histomorphometric analysis of new bone obtained by osteogenic periosteal distraction in ovariectomized rabbits. Oral Surg Oral Med Oral Pathol Oral Radiol 2012;113:472-479.

31. Kim UK, Park SJ, Seong WJ, Heo J, Hwang DS, Kim YD, Shin SH, Kim GC. Expression of TGF-beta1, osteonectin, and BMP-4 in mandibular distraction osteogenesis with compression stimulation: reverse transcriptase-polymerase chain reaction study and biomechanical test. J Oral Maxillofac Surg 2010;68:2076-2084.

32. Ma L, Zheng LW, Sham MH, Cheung LK. Effect of nicotine on gene expression of angiogenic and osteogenic factors in a rabbit model of bone regeneration. J Oral Maxillofac Surg 2010;68:777-781. 
33. Matsubara H, Hogan DE, Morgan EF, Mortlock DP, Einhorn TA, Gerstenfeld LC. Vascular tissues are a primary source of BMP2 expression during bone formation induced by distraction osteogenesis. Bone 2012;51:168-180.

34. Choi IH, Ahn JH, Chung CY, Cho TJ. Vascular proliferation and blood supply during distraction osteogenesis: a scanning electron microscopic observation. J Orthop Res 2000;18:698-705.

35. Ziros PG, Gil AP, Georgakopoulos T, Habeos I, Kletsas D, Basdra EK, Papavassiliou AG. The bone-specific transcriptional regulator Cbfa1 is a target of mechanical signals in osteoblastic cells. The Journal of biological chemistry 2002;277:23934-23941.

36. Kanno T, Takahashi T, Ariyoshi W, Tsujisawa T, Haga M, Nishihara T. Tensile mechanical strain up-regulates Runx2 and osteogenic factor expression in human periosteal cells: implications for distraction osteogenesis. J Oral Maxillofac Surg 2005;63:499-504.

37. Kim IS, Song YM, Cho TH, Kim JY, Weber FE, Hwang SJ. Synergistic action of static stretching and BMP-2 stimulation in the osteoblast differentiation of $\mathrm{C} 2 \mathrm{C} 12$ myoblasts. J Biomech 2009;42:2721-2727.

38. Tselentakis G, Kitano M, Owen PJ, Kuiper JH, Richardson JB, Evans GA. The behaviour of the periosteum during callotasis. J Pediatr Orthop B 2003;12:277-283.

39. Allen MR, Hock JM, Burr DB. Periosteum: biology, regulation, and response to osteoporosis therapies. Bone 2004;35:1003-1012.

40. Takiguchi S, Kuboyama, N., Kuyama, K., Yamamoto, H., Kondoh, T. Experimental Study of Bone Formation Ability with the Periosteum on Rat Calvaria. J Hard Tissue Biology 2009;18:149-160.

41. Saulacic N, Schaller B, Bosshardt DD, Buser D, Jaun P, Haeniwa H, lizuka T. Periosteal distraction osteogenesis and barrier membrane application: an experimental study in the rat calvaria. J Periodontol 2012;83:757-765.

42. Matsuo A, Chiba H, Takahashi H, Toyoda J, Hasegawa O, Hojo S. Bone quality of mandibles reconstructed with particulate cellular bone and marrow, and platelet-rich plasma. J Craniomaxillofac Surg 2011;39:628-632.

43. Wiren KM, Hashimoto JG, Semirale AA, Zhang XW. Bone vs. fat: embryonic origin of progenitors determines response to androgen in adipocytes and osteoblasts. Bone 2011;49:662-672. 
44. Yoshiko Y, Oizumi K, Hasegawa T, Minamizaki T, Tanne K, Maeda N, Aubin JE. A subset of osteoblasts expressing high endogenous levels of PPARgamma switches fate to adipocytes in the rat calvaria cell culture model. PLoS One 2010;5:e11782.

45. Meyer U, Meyer T, Wiesmann HP, Kruse-Losler B, Vollmer D, Stratmann U, Joos U. Mechanical tension in distraction osteogenesis regulates chondrocytic differentiation. Int J Oral Maxillofac Surg 2001;30:522-530.

46. Furuta N, Yoshioka I, Fukuizumi T, Tominaga K, Nishihara T, Fukuda J. Morphological features of cartilage observed during mandibular distraction in rabbits. Int $\mathrm{J}$ Oral Maxillofac Surg 2007;36:243-249.

47. Fujii T, Ueno T, Kagawa T, Sugahara T, Yamamoto T. Immunohistochemical analysis of Sox9 expression in periosteum of tibia and calvaria after surgical release of the periosteum. Acta histochemica 2005;106:427-437.

48. Govender S, Csimma C, Genant HK, Valentin-Opran A, Amit Y, Arbel R, et al. Recombinant human bone morphogenetic protein-2 for treatment of open tibial fractures: a prospective, controlled, randomized study of four hundred and fifty patients. J Bone Joint Surg Am 2002;84A:2123-2134.

49. Garrison KR, Donell S, Ryder J, Shemilt I, Mugford M, Harvey I, et al. Clinical effectiveness and cost-effectiveness of bone morphogenetic proteins in the non-healing of fractures and spinal fusion: a systematic review. Health Technol Assess 2007;11:1-150, iii-iv.

50. Albers CE, Hofstetter W, Sebald HJ, Sebald W, Siebenrock KA, Klenke FM. L51P - A BMP2 variant with osteoinductive activity via inhibition of Noggin. Bone 2012;51:401-406. 


\section{FIGURE LEGENDS}

FIGURE 1. Protocol of periosteal distraction applied in two groups of animals.

FIGURE 2. (A) Intraoperative view of the area following flap elevation and placement of distraction device. (B) The periosteum and (C) the skin are closed with interruptive sutures in two layers.

FIGURE 3. Micro-CT images and the corresponding transversal histological sections of the calvarium illustrating central the region of the distraction device in Group I after distraction period (A, B, C), 2-week consolidation period (D, E, F) and 2-month consolidation period (G, H, I). (A) Micro-CT image illustrates old bone and new bone underneath the distraction device. (B) Overview showing the patch-wise deposition of new bone (NB) on top of the old calvarial bone (CB) after distraction period. Old bone consists of tabula interna, tabula externa, and intervening mature bone marrow with signs of bone remodeling. (C) Fine trabecular network of new woven bone (WB) is highly vascularized. Orbicular structures are observed at the leading edge of bone formation facing the soft connective tissue (arrowheads). (D, G) Micro-CT images during consolidation period match the histological observations. (E) At 2-week consolidation period, layer of new bone with vascularized bone marrow is present along the calvarial bone. (F) New woven bone is reinforced by parallelfibered bone with immature bone marrow (BM). Osteoblasts and osteoid $\left(^{*}\right)$ are clearly visible at the leading front of bone formation. (H) Overview showing a contiguous layer of new, mature bone deposited on the calvarial bone at 2-month consolidation period. Fat tissue is present within the bone cavities and the distraction gap. (I) Osteoid layers (arrows) cover the surface of the newly formed bone.

FIGURE 4. Micro-CT images and the corresponding transversal histological sections of the calvarium illustrating central region of the distraction device in Group II after distraction period (A, B, C), 2-week consolidation period (D, E, F) and 2-month consolidation period (G, H, I). (A) Micro-CT image illustrates old bone and new bone within the distraction gap. (B) Overview of new bone (NB) formed along the periosteum with large bone marrow cavities after distraction period. Elongated trabucules of woven bone are oriented parallel to the distraction vector. (C) Osteoblasts (arrows) facing the even surface of new bone are indicating ongoing bone apposition. (D, G) During 
consolidation period, micro-CT images match the histological observations. (E) New bone with irregular bone islets and immature bone marrow associated with calvarial bone is observed at 2week consolidation period. (F) Layer of new bone has irregular, flat contour (arrowheads) deprived from the periosteum. Osteoid and osteoblasts (arrows) are visible on the surface of the bone cavity with immature bone marrow (BM). (H) At 2-month consolidation period, mature bone is formed along the periosteum with irregular bone sprouts within the distraction gap, rich in fat tissue. The height of the new bone was about three times greater than that of the old calvarial bone. (I) Superficial layer of mature bone (arrows) is indicative of ongoing bone formation.

FIGURE 5. Periosteal distraction osteogenesis induces the expression of transcripts encoding tissue-specific genes. The level of BMP2 mRNA in Group I significantly increased in bone at 2-week consolidation period in comparison to Group II and within Group I, compared to mid-distraction, enddistraction and 2-month consolidation period $\left({ }^{*} p<0.01\right)$. Increased values of RUNX2, SPARC and SOX9 mRNA were observed in Group II at mid-distraction period compared to 1-week consolidation period $\left({ }^{* *} p<0.05\right)$. There were no differences between the groups in the level of transcripts in soft tissue. The expression of SPARC mRNA was increased in Group I and Group II at mid-distraction period and SOX9 mRNA in Group II at mid-distraction period $\left({ }^{* *} p<0.05\right)$. Periosteal distraction osteogenesis induces the expression of transcripts encoding tissue-specific genes. In periosteum, value of RUNX2 mRNA in Group II was significantly increased at mid-distraction period in comparison to Group I ( $\left.{ }^{* *} p<0.05\right)$ and within the group, compared to 2 -months consolidation period $\left({ }^{*} p<0.01\right)$. 